\title{
PRÁTICAS INTEGRATIVAS E COMPLEMENTARES EM SAÚDE
}

Daniel Miele Amado, Fernanda Elizabeth Sena Barbosa, Layza Nogueira Dias Dos Santos, Lorena Toledo de Araújo Melo, Paulo Roberto Sousa Rocha, Rafael Dall Alba

\section{RESUMO}

Este artigo tem como objetivo analisar as experiências submetidas ao prêmio APS Forte de iniciativas de Práticas Integrativas Complementares ICS que demonstram como as mesmas estão inseridas e incorporadas na APS.

Palavras-chave: Atenção Primária à Saúde; Acesso; Prêmio APS.

\section{ABSTRACT}

This article aims to analyze the experiences submitted to the APS Forte Award for ICS Complementary Integrative Practices initiatives that demonstrate how they are inserted and incorporated into PHC.

Keywords: Prmary Health Care; Access; PHC Prize.
Revista da Rede APS 2020 Publicada em: 04/09/2020 DOI:10.14295/aps.v2i3.150

Daniel Miele Amado Fernanda Elizabeth Sena Barbosa

Layza Nogueira Dias Dos Santos Lorena Toledo de Araújo Melo Paulo Roberto Sousa Rocha (Coordenação Nacional de Práticas Integrativas e Complementares em Saúde DESF/SAPS/Ministério da Saúde)

Rafael Dall Alba

(Organização Pan-Americana da Saúde OPAS/OMS)

Correspondência para:

Daniel Miele Amado daniel.amado@saude.gov.br 


\section{INTRODUÇÃO}

A Política Nacional de Práticas Integrativas e Complementares no SUS - PNPIC, é uma política pública que se estabeleceu a partir de demandas sociais recorrentes manifestadas nas diversas Conferências Nacionais de Saúde, e das diretrizes da Organização Mundial Saúde - OMS. (BRASIL, 2006).

As Práticas Integrativas e Complementares em Saúde - PICS, como são conhecidas no Brasil, fazem parte das práticas denominadas pela OMS de Medicinas Tradicionais, Complementares e Integrativas - $\mathrm{MTCl}$. As $\mathrm{MTCl}$ se referem a um amplo conjunto de práticas de cuidado em saúde que podem variar de país a país e das práticas instituídas no sistema convencional de saúde. As Medicinas Tradicionais, de alguns países, são adotados por outros como Medicinas Complementares ou Integrativas, como exemplo da Medicina Tradicional Chinesa ou o Ayurveda, que são amplamente utilizadas fora de seus países de origem. (WHO, 2019).

A OMS, desde a década de 70 reconhece a importância das $\mathrm{MTCl}$ para assegurar o acesso da população mundial a serviços de saúde, principalmente de atenção primária, e orienta aos Estados Membros para que reconheçam, fomentem e normatizem as $\mathrm{MTCl}$ em seus sistemas nacionais de saúde. A Estratégia da OMS sobre Medicina Tradicional 2002-2005 preconiza políticas de integração entre a medicina convencional e outras diferentes racionalidades e práticas nos sistemas nacionais de saúde considerando a segurança, a eficácia e a qualidade dessas práticas, disponibilizando orientações acerca da legislação; acesso às práticas; e uso racional por profissionais e consumidores (WHO, 2002). As Estratégias das Medicinas Tradicionais da OMS para 2014-2023 abordam normas, padrões, informações e dados confiáveis para apoiar os Estados Membros no provimento de serviços de $\mathrm{MTCl}$ seguros, qualificados e eficazes para integração adequada nos sistemas de saúde, visando o alcance da cobertura universal de saúde e os Objetivos de Desenvolvimento Sustentável (ODS). (WHO, 2013). A OMS publicou em 2019 o
Global Report, relatório que analisa o avanço global das $\mathrm{MTCl}$ nas últimas décadas, a partir das contribuições de 179 países Estados Membros da OMS. Até 2018, um total de 98 EstadosMembros haviam desenvolvido políticas nacionais de $\mathrm{MTCl}$, o que representa $50 \%$ dos países membros da OMS, 109 haviam publicado leis ou regulamentações nacionais e 124 haviam implementado regulamentações sobre medicamentos fitoterápicos. (WHO, 2019).

Em termos de oferta das $\mathrm{MTCl}$ no mundo, na Europa, o percentual de indivíduos que utilizaram alguma vez a $\mathrm{MTCl}$ representa $31 \%$ na Bélgica, $75 \%$ na França, $48 \%$ na Austrália e no Canadá estima-se que $70 \%$ da população fazem uso de alguma dessas práticas (SOUSA et al, 2012).

Nesse contexto, o Brasil está na vanguarda entre os Estados Membros da OMS em termos de avanço tanto do ponto de vista da regulamentação, construção de políticas em $\mathrm{MTCl}$, como na integração das $\mathrm{MTCl}$ no sistema nacional de saúde. No âmbito do Sistema Único de Saúde (SUS), as PICS foram institucionalizadas no ano de sua constituição em 1988, por meio das resoluções da Comissão Interministerial de Planejamento e Coordenação, que instituíram serviços de acupuntura, homeopatia, plantas medicinais e termalismo. Enquanto política nacional, com diretrizes para os três entes federados, se instituiu a partir da Política Nacional de Práticas Integrativas e Complementares no SUS, por meio da Portaria GM/MS no 971, de 3 de maio de 2006. (BRASIL, 2006).

A PNPIC quando publicada, contemplou diretrizes e responsabilidades institucionais para oferta de serviços e produtos da homeopatia, medicina tradicional chinesa/acupuntura, plantas medicinais e fitoterapia, medicina antroposófica e termalismo social/crenoterapia, além de estimular a ampliação destas práticas em diversos municípios brasileiros. Em 27 de março de 2017, a PNPIC foi ampliada em outras 14 práticas a partir da publicação da Portaria Ministerial no 849, a saber: arteterapia, ayurveda, biodança, dança circular, meditação, 
musicoterapia, naturopatia, osteopatia, quiropraxia, reflexoterapia, reiki, shantala, terapia comunitária integrativa e yoga, totalizando 19 práticas. (BRASíLIA, 2017). E, em 21 de março de 2018, com a publicação da Portaria Ministerial $n^{\circ}$ 702, foram incluídas outras dez práticas na PNPIC, sendo: apiterapia, aromaterapia, bioenergética, constelação familiar, cromoterapia, geoterapia, hipnoterapia, imposição de mãos, ozonioterapia e terapia de florais. (BRASÍLIA, 2018). Tais práticas ampliam as abordagens de cuidado e as possibilidades terapêuticas para os usuários, garantindo uma maior integralidade e resolutividade da atenção à saúde. (BRASIL, 2006).

A implantação da PNPIC cumpre com as recomendações da OMS, além das diversas conferências nacionais de saúde, e assembleias mundiais, promovendo e integrando a oferta das PICS no SUS. Além disso, protagoniza e embasa discussões com as diversas áreas que dialogam com esta política, assim como instituições responsáveis pelo desenvolvimento de legislação/normatização para oferta de serviços e produtos de qualidade, propiciando o desenvolvimento dos conhecimentos na área. (BRASIL, 2006).

Nesta perspectiva, a PNPIC é transversal em suas ações no SUS e está presente em todos os níveis de atenção, em especial na Atenção Primária à Saúde (APS), e com grande potencial de atuação nas Redes de Atenção à Saúde (RAS). (BRASIL, 2006). Dados do ano de 2018, mostram que as PICS estiveram presentes em 16.007 serviços de saúde do SUS, sendo 14.508 (90\%) da Atenção Primária à Saúde (APS), distribuídos em 4.159 municípios (74\%) - APS e média e alta complexidade - e em todas das capitais (100\%).Foram ofertados 989.704 atendimentos individuais, 81.518 atividades coletivas com 665.853 participantes e 357.155 procedimentos em PICS. Já parciais para o ano de 2019, as PICS estiveram presentes em 17.335 serviços de saúde do SUS, sendo 15.601 (90\%) da Atenção Primária à Saúde (APS), distribuídos em 4.296 municípios (77\%) - APS e média e alta complexidade - e em todas das capitais (100\%).Foram ofertados 693.650 atendimentos individuais, 104.531 atividades coletivas com 942.970 participantes e 628.239 procedimentos em PICS.(Fonte: SISAB/DATASUS para o ano de 2018 e parciais para o ano de 2019).

Além disso, o tema de PICS está inserido no PMAQ-AB, Programa Nacional de Melhoria do Acesso e da Qualidade da Atenção Básica, lançado em 2011 pelo Ministério da Saúde, programa que tem como objetivo melhorar o acesso e a qualidade dos serviços da APS. (BRASIL, 2015). O primeiro ciclo do PMAQ foi realizado entre os anos de 2011 e 2012 , o $2^{\circ}$ ciclo entre 2013 e 2014 e $3^{\circ}$ ciclo entre 2015 e 2019.

Desde $01^{\circ}$ ciclo, foi possível observar os resultados positivos em relação a oferta de PICS, que permitiram não só a ampliação dos parâmetros sobre o tema no $2^{\circ}$ ciclo, como a alocação de recursos voltados à pesquisa e fortalecimento dos serviços de PICS. O $1^{\circ}$ e $2^{\circ}$ ciclo do PMAQ demonstraram que $20 \%$ das equipes de saúde ofertavam alguma prática integrativa. Já o $3^{\circ}$ ciclo apontou que $31.48 \%$ das equipes ofertam PICS. (BRASIL, 2019).

Os dados sugerem que as PICS, enquanto forma de cuidado em saúde, são ofertadas cada vez mais pelos profissionais e serviços e por mais municípios, em todo o território nacional, sendo mais integradas por parte dos profissionais de saúde em suas abordagens clínicas.

Assim, este artigo tem como objetivo analisar as experiências submetidas ao prêmio APS Forte de iniciativas de PICS que demonstram como as mesmas estão inseridas e incorporadas na APS.

\section{Metodologia}

O presente estudo é de natureza descritivo qualitativa. Dentro do processo de avaliação dos mais de 1200 relatos, 80 foram classificados como sendo de PICS, por enfatiza-las na descrição. No entanto, observou-se que um total de 143 experiências apresentavam PICS em seus relatos e foram classificados em outros temas, totalizando 223 experiências com PICS. Para análise do material foram realizadas leituras das experiências e com o auxílio da metodologia de nuvem de palavras foi composto o quadro de temas mais recorrentes 
por meio da ferramenta ATLAS TI 7. O tamanho de cada palavra indica sua frequência, admitida como proxy da relevância de determinada temática (RAMSDEN, 2008). na totalidade das experiências. Foram categorizadas as principais práticas integrativas, interfaces com outros serviços, áreas e inovação no cuidado.

\section{RESULTADOS}

Dentre as 80 experiências selecionadas, várias PICS foram destacadas. Alguns relatos trouxeram experiências com mais de uma prática integrativa para uma condição ou situação específica em saúde. Assim, práticas como plantas medicinais e fitoterapia, auriculoterapia, meditação e acupuntura apareceram em vários relatos, sendo aplicadas isoladamente e/ou conjuntamente com outras práticas. A tabela abaixo traz a lista de PICS com o número de relatos em que elas foram apontadas, agrupadas de acordo com a portaria que a institui na PNPIC.

Tabela 1 - Práticas Integrativas

\begin{tabular}{|c|c|c|}
\hline PORTARIA PNPIC & PRÁTICA INTEGRATIVA & TOTAL \\
\hline \multirow{6}{*}{2006} & MTC-Auriculoterapia & 28 \\
\hline & MTC - Tai Chi Chuan e Qigong & 5 \\
\hline & MTC - Acupuntura & 6 \\
\hline & MTC - sem especificar & 2 \\
\hline & Homeopatia & 1 \\
\hline & Plantas medicinais e fitoterapia & 19 \\
\hline \multirow{9}{*}{2017} & Arteterapia & 2 \\
\hline & Dança circular & 1 \\
\hline & Meditação & 9 \\
\hline & Musicoterapia & 1 \\
\hline & Naturopatia & 1 \\
\hline & Reiki & 9 \\
\hline & Shantala & 2 \\
\hline & Terapia comunitária & 1 \\
\hline & Yoga & 4 \\
\hline \multirow{6}{*}{2018} & Aromaterapia & 3 \\
\hline & Bioenergética & 2 \\
\hline & Constelação familiar & 3 \\
\hline & Cromoterapia & 1 \\
\hline & Geoterapia & 1 \\
\hline & Terapia de florais & 3 \\
\hline
\end{tabular}

Interfaces com outros serviços e áreas

Os 80 relatos classificados como PICS apresentavam relação com diversas áreas e programas de saúde, como saúde do trabalhador, Programa Saúde na Escola e assistência farmacêutica, entre outras, conforme listado abaixo: 
Tabela 2 - Áreas e programas relacionados com os relatos

\begin{tabular}{|c|c|c|}
\hline $\begin{array}{c}\text { Assistência } \\
\text { farmacêutica }\end{array}$ & $\begin{array}{c}\text { Doenças crônicas não } \\
\text { transmissíveis }\end{array}$ & Regulação \\
\hline Saúde do trabalhador & Educação permaneñão da saúde \\
\hline Saúde mental & Programa de tabagismo & $\begin{array}{c}\text { Formação em saúde e Relação } \\
\text { ensino-serviço }\end{array}$ \\
\hline $\begin{array}{c}\text { Programa Saúde na } \\
\text { Escola }\end{array}$ & Agentes Comunitários de Saúde & Saúde a população de rua \\
\hline
\end{tabular}

Além disso, as experiências demonstraram a utilização das práticas integrativas em expressivo número de condições de saúde como forma de cuidado em situações como diabetes, fibromialgia, dor crônica, problemas mentais, ansiedade e estresse. As condições de saúde em que as PICS foram utilizadas:

Tabela 3 - PICS e condições de saúde

\begin{tabular}{|c|c|}
\hline PRÁTICA INTEGRATIVA & INOVAÇÃO NO CUIDADO \\
\hline \multirow{3}{*}{ PICS em Geral } & Saúde do trabalhador \\
\hline & Fibromialgia \\
\hline & Dor \\
\hline Plantas medicinais e fitoterapia & Hipertensão \\
\hline \multirow{7}{*}{ Auriculoterapia } & Fibromialgia \\
\hline & Dor Crônica \\
\hline & Sofrimento \\
\hline & Tabagismo \\
\hline & Estresse \\
\hline & Ansiedade \\
\hline & Saúde do trabalhador \\
\hline \multirow{5}{*}{ Yoga } & Controle da pressão arterial \\
\hline & Ansiedade \\
\hline & Sono \\
\hline & Depressão \\
\hline & Equilíbrio emocional e mental \\
\hline Acupuntura & Diabetes \\
\hline Meditação & Transtornos mentais \\
\hline
\end{tabular}

Complementarmente, as PICS foram relatadas como forma de cuidado para outras situações, a exemplo da melhora da qualidade do sono, tratamento para enxaqueca, síndrome do pânico, redução da obesidade, além de aspectos relacionados à socialização, vulnerabilidade social, equilíbrio emocional e dependência química, como consta a seguir:

- Tratamento e prevenção de dores no corpo;

(c) 
- Tratamento de problemas osteomusculares e articulares;

- Desenvolvimento da socialização;

- Redução da ansiedade;

- Controle do estresse;

- Melhora da qualidade de sono;

- Tratamento para enxaqueca;

- Tratamento da depressão e síndrome do pânico;

- Tratamento nos quadros de hipertensão e diabetes;

- Tratamento de doenças crônicas do aparelho respiratório, cardiovascular e nervoso;

- Redução da obesidade;

- Tratamento do tabagismo;

- Complementar ao tratamento das dependências químicas;

- Melhorar a qualidade de pessoas em vulnerabilidade social;
- Aumento na produtividade;

- Melhora no desenvolvimento escolar;

- Redução de sintomas de agitação;

- Tratamento de feridas utilizando: redução do tempo de cicatrização, redução de antiinflamatórios;

- Melhora no foco/atenção;

- Redução da fome/aumento da saciedade;

- Melhora do equilíbrio emocional;

- Promoção do equilíbrio da circulação de sangue e de energia corporal;

- Melhoria no quadro geral da dor;

- Diminuição do uso excessivo de medicamentos;

- Percepção de melhora da gastrite, labirintite, sinusite e otite;

A nuvem de palavras apresentou a seguinte disposição:

Figura 1 - Nuvem de palavras

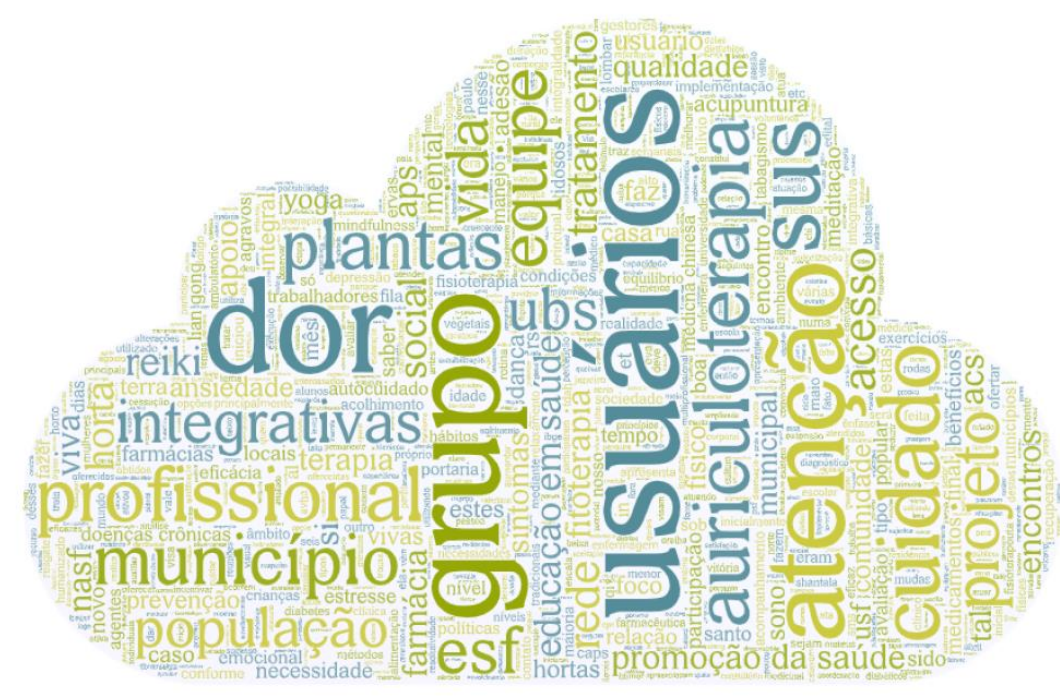


Pode-se observar que algumas palavras são de grande destaque, como dor, grupos, usuários, atenção, cuidado e auriculoterapia.
Quando foram analisadas a presença das PICS em outras categorias de experiências, observouse expressiva utilização das PICS para as mais diversas condições, associadas aos seguintes campo da saúde:

Tabela 4 - Número de experiências por campo da saúde

\begin{tabular}{|c|c|}
\hline Campo da Saúde & Número de experiências \\
\hline Saúde mental & 22 \\
\hline Processo de trabalho & 19 \\
\hline Promoção e prevenção da Saúde & 17 \\
\hline Atividade Física e Academia da Saúde & 12 \\
\hline Saúde Materno-infantil & 11 \\
\hline Áreas remotas ou vulnerabilidade & 11 \\
\hline Doenças Crônicas Não Transmissíveis & 8 \\
\hline Alimentação e Nutrição & 6 \\
\hline Ampliação e flexibilização de horários & 6 \\
\hline Capacidades Humanas & 6 \\
\hline Tecnologia da Informação & 5 \\
\hline Saúde do Idoso & 5 \\
\hline Flexibilização de agendas & 4 \\
\hline Saúde Bucal & 4 \\
\hline Saúde da Mulher & 3 \\
\hline Saúde do Adolescente & 2 \\
\hline Contratualização & 1 \\
\hline Saúde do Homem & 1 \\
\hline Imunização & 1 \\
\hline Ampliação de cobertura da ESF & 1 \\
\hline IST & 1 \\
\hline
\end{tabular}

\section{DISCUSSÃo}

A PNPIC advém de movimentos do território pela busca de formalizar o que já estava sendo realizado nos municípios. Assim, anterior à publicação da PNPIC, já era sabido que várias PICS eram ofertadas nos serviços públicos de saúde (SANTOS, 2011; BRASIL, 2006), como uma forma de cuidado que buscava olhar o indivíduo integralmente. A institucionalização de outras abordagens de cuidado, com a criação da PNPIC foi um importante avanço para a consolidação do SUS.

A presença expressiva de experiências com as PICS nos relatos submetidos ao prêmio APS forte, cerca de $18 \%$, demonstra que as PICS têm sido integradas como um cuidado inovador para diferentes questões da complexidade do cuidado na APS. Essa inovação se dá majoritariamente por iniciativa municipal considerando que as PICS não possuem um financiamento específico federal no âmbito da APS.

Desta forma, os municípios estão integrando outras formas de cuidar da população, diante de um contexto atual de transição epidemiológica e etária, com aumento da prevalência de DCNT, de transtornos mentais e obesidade, de envelhecimento da população, uso excessivo de medicamentos e fragmentação da atenção à 
saúde. No manejo de usuários com doenças crônicas, o autocuidado é etapa imprescindível para adoção de estilo e prática de vida mais saudáveis. Nesse contexto as PICS são importantes aliadas, contribuindo com a promoção para o autocuidado, até o manejo clínico das DCNT, potencializando o tratamento por meio de seus benefícios (KIM, 2018, FALKENBERG,2018).

Um destaque a ser realizado é a resposta das PICS frente a crescente demanda relacionada a dor crônica que possui uma prevalência aproximada no Brasil de $30 \%$ da população (VASCONCELOS, 2018). O que é possível observar é que o modelo de cuidado ofertado pela rede de serviços de saúde tem tentado se adaptar à mudança no perfil das necessidades de saúde da população, procurando um modelo de cuidado centrado na pessoa e não na doença, centrado na família e na comunidade, um modelo que considere e atue na complexidade biopsicossocial. Práticas integrativas, como meditação, yoga, plantas medicinais e práticas corporais da Medicina Tradicional Chinesa, demonstram eficácia do manejo da dor (VEEHOF, 2011; CRAMER, 2013; BRASIL, 2015; ZHANG, 2017).

A saúde mental foi outro campo que pode ser observado sua ampla utilização nas experiências analisadas. As relações de cuidado, a partir de estratégias de acolhimento, vínculo, autonomia e responsabilização no cuidado, funcionam como dispositivos eficazes para a promoção da saúde mental e para o desenvolvimento de práticas integrais. Diversas PICS, a exemplo da meditação, acupressão, aromaterapia e auriculoterapia, são eficazes nos casos de ansiedade, depressão e estresse, impactando positivamente na regulação e melhoria do estado mental de indivíduos (KUYKEN, 2015; DOREEN, 2016; KWON, 2020).

A maior parte dos serviços de PICS são ofertadas na APS, o que representa avanços na implementação da PNPIC, visto que a inserção prioritária de PICS neste nível de atenção é objetivo desta política (BRASIL, 2006). Esta inserção corrobora com o fato das PICS e a APS compartilharem afinidades como a abordagem familiar e comunitária, centramento nos sujeitos em seus contextos sociais e familiares, adequação sócio-cultural, construção de vínculos terapêuticos, dentre outros (TESSER; SOUSA; 2012).

Por estar mais próximo do cotidiano de indivíduos e famílias, a APS demanda de tecnologias leves de elevada complexidade para solucionar os principais problemas sanitários, sociais e ambientais das comunidades assistidas (MERHY, 2002). Tal complexidade demanda ampliação das abordagens de cuidado e de opções terapêuticas para possibilitar intervenções mais resolutivas e eficazes. Esta é uma possível justificativa do uso de diversas PICS identificadas nas experiências analisadas e que demonstraram oferta de mais de 5 práticas em diferentes situações, como atendimento à demanda espontânea, atendimento coletivo, grupos terapêuticos, fila de espera para especialistas como ortopedia e fisioterapia, e assim, podendo contribuir para uma melhor resolutividade da APS.

A atuação multiprofissional é uma característica da oferta de PICS, com participação da equipe mínima, mas também de outros profissionais, demonstrando que essas práticas de cuidado são compartilhadas, o que pode contribuir para a descentralização do cuidado pelas consultas individuais do profissional médico e de enfermagem. É possível, que ao racionalizar esses saberes para condições mais específicas, haja uma ampliação do acesso ao cuidado na UBS e, assim, uma maior resolutividade de toda a equipe. Um fato observado nos relatos das experiências, é a relação de utilização e consumo das ações das Unidades de Saúde pelos usuários que passam por uma ressignificação, mudando o perfil de utilização, e como fator recorrente, um aumento de vínculo com o serviço de saúde. Essa mudança também pode ocorrer com os profissionais de saúde, que se beneficiam com as PICS e o autocuidado.

Um ponto importante a ser ressaltado é que as PICS atuam igualmente para o empoderamento do sujeito, favorecendo a percepção dos processos de adoecimento e de cuidado, seja nos aspectos individuais, coletivos ou sociais, 
com a aquisição de ferramentas que possibilitam o autocuidado, promovendo desde a valorização dos saberes tradicionais, como o uso de plantas medicinais, até a apropriação da comunidade em técnicas simples como shantala, massagem, reiki e meditação. 0 envolvimento da comunidade em sua prática de cuidado, tem gerado a construção de redes cuidadoras e grupos de voluntários que cuidam da própria comunidade. Esse processo de empoderamento individual e coletivo possibilita o uso mais racional das ações, serviços de saúde e insumos, desprivilegiando a cultura de medicalização e de utilização exagerada e indevida de exames e procedimentos, o que impacta diretamente nos custos do sistema de saúde.

Com base nas experiências analisadas, as farmácias vivas e hortas de plantas medicinais foram experiências amplamente relatadas. Neste contexto, estes serviços têm sido apresentados como uma forma de acesso a medicamentos no sistema de saúde (ANVISA, 2011; ANVISA 2016; ANVISA 2018), além de envolver a comunidade no compartilhamento de saberes, ações de educação em saúde, socialização, atividades intergeracionais e intersetoriais (COSTA, 2015).

A atuação intersetorial das PICS principalmente nas escolas, tem se apresentado presente em diversos relatos, não apenas como uma intervenção de saúde, mas como uma melhoria da qualidade de vida dos alunos, cuidado de saúde mental, que tem gerado, segundo os relatos, progresso no desenvolvimento escolar.

A capacitação de mais profissionais em PICS é relatada pelas experiências como uma das dificuldades de expansão dessas práticas, de forma a garantir a oferta desse cuidado para mais pessoas. Isso corrobora com a literatura que reconhece que a formação em PICS no Brasil é insuficiente e difusa, que estas práticas estão sendo gradativamente inseridas nas graduações dos profissionais de saúde, porém essa formação ainda é insuficiente. Quanto aos profissionais que estão em serviço, existem ações de formação ofertadas pelo MS, sendo a maior parte dos cursos de carácter introdutório e um semipresencial em Auriculoterapia, assim como outras ofertas são realizadas por secretarias de saúde (TESSER, 2018). A formação de cerca de 10 mil profissionais de saúde em Auriculoterapia em polos espalhados por todas as regiões do país pode ter contribuído para esta ser a prática mais relatado no Prêmio.

A melhoria da qualidade de vida é uma expressão recorrente nos relatos, assim como a socialização, mostrando que as PICS têm sido utilizadas de forma mais ampla do que apenas para o tratamento de queixas específicas. As PICS se relacionam com uma dimensão de viver do sujeito, não centradas nas patologias instaladas ou com probabilidades de serem contraídas, tampouco centra-se nos possíveis métodos de controle e prevenção das mesmas (LUZ, 2013). O status moderno das doenças e do adoecer adquire uma mudança do "mal-estar" individual para um mal estar coletivo podendo ser visto como um fenômeno de natureza tanto sanitária como cultural, que tem suas raízes não apenas nas condições de trabalho do capitalismo globalizado, mas na própria transformação recente da cultura que é seu fruto (LUZ, 1997). Portanto as PICS por tensionarem a estrutura hermética biomédica empregada nos serviços de saúde, deslocam também a epistemologia do cuidado em si, ampliando não só as práticas clínicas, fazendo alusão a uma caixa de ferramentas empregadas no cuidado mas também as "lentes" empregadas nesse cuidado, abrangendo outras racionalidades médicas diversificando as abordagens terapêuticas, anatômicas, diagnósticas e fisiológicas e por fim podendo modificar a própria cosmologia do que tange o cuidado e a saúde.

\section{CONSIDERAÇÕES FINAIS}

A construção de um sistema público e universal de saúde apresenta diversos desafios, mesmo estando em consonância com estratégias defendidas pela OMS, que o reconhece como principal forma de garantir a saúde da população. A estruturação de um sistema universal que busca a integralidade e a equidade para um território, nas dimensões do Brasil, necessita de um modelo eficiente, com atuação 
intersetorial e que empodere sujeitos e comunidades no desempenho dos processos que interagem com os diversos determinantes sociais da saúde. É neste ponto que as PICS se inserem no contexto do SUS.

As PICS têm demonstrado, pelos relatos apresentados, que ampliam o acesso da população ao cuidado, de forma multiprofissional, equalizando o acesso às consultas médicas, e assim, também amplia o acesso a esse profissional. A APS, ao incorporar as PICS, se torna mais resolutiva para diversas condições, se destacando as dores, as DCNT e a saúde mental, promovendo um uso mais racional de serviços, ações de saúde e insumos. Para além das condições de saúde, essas práticas ampliam a qualidade de vida da população, aprofunda o vínculo com o serviço, ressignificando a utilização da unidade de saúde. Essas práticas, ainda, empoderam os sujeitos no seu autocuidado, valorizam os saberes tradicionais, e criam redes cuidadoras nos territórios.

Apesar da diversidade de racionalidades e de possibilidades terapêuticas, a práxis das PICS apresenta como característica o foco do cuidado na pessoa e não na doença, a adaptação do cuidado ao usuário e seu contexto.

Nesta perspectiva, as PICS são relatadas nas diversas experiências, como inovação no cuidado, ampla adesão da população e de profissionais de saúde, fortalecendo os atributos essenciais da APS, contribuindo para um sistema de saúde mais resolutivo, sustentável e racional. 


\section{NOTAS E REFERÊNCIAS}

BRASIL. Agência Nacional de Vigilância Sanitária. Formulário de Fitoterápicos da Farmacopeia Brasileira [Livro]. - Brasília : ANVISA, 2018. - 1a Edição : Vol. Primeiro Suplemento : p. 160.

Agência Nacional de Vigilância Sanitária. Memento Fitoterápico da Farmacopeia Brasileira [Livro]. - Brasília : ANVISA, 2016. - 1a. Ed. : p. 117.

Agência Nacional de Vigilância Sanitária. Formulário de Fitoterápicos da Farmacopeia Brasileira [Livro]. - Brasília : ANVISA, 2011. - p. 126.

Ministério da Saúde. Monografia da espécie Salix alba (SALGUEIRO BRANCO). Brasília, 2015. Disponível em: https://www.saude.gov.br/images/pdf/2017/setembro/11/Monografia-Salix-alba.pdf Acesso em 16 abr 2020.

Ministério da Saúde. Portaria no 702, de 21 de março de 2018. Altera a Portaria de Consolidação no 2/GM/MS, de 28 de setembro de 2017, para incluir novas práticas na Política Nacional. Diário Oficial da União. 22 mar 2018

Ministério da Saúde. Portaria no 849, de 27 de março de 2017. Inclui a Arteterapia, Ayurveda, Biodança, Dança Circular, Meditação, Musicoterapia, Naturopatia, Osteopatia, Quiropraxia, Reflexoterapia, Reiki, Shantala, Terapia Comunitária Integrativa e Yoga à Política Nacional de Práticas Integrativas e Complementares. Diário Oficial da União. 28 mar 2017

Ministério da Saúde. Portaria no 1.645, de 02 de outubro de 2015. Dispõe sobre o Programa Nacional de Melhoria do Acesso e da Qualidade da Atenção Básica (PMAQ-AB). 2015. Disponível em: http://bvsms.saude.gov.br/bvs/saudelegis/gm/2015/prt1645 0110 2015.html.

Ministério da Saúde. Secretaria de Atenção Primária à Saúde. Programa Nacional de Melhoria do Acesso e da Qualidade da Atenção Básica (PMAQ). 3 ciclo. Retratos da APS - relatório da avaliação externa do PMAQ 3o ciclo. Disponível em: https://retratos.navi.ifrn.edu.br/\#cards-category Acesso em: 09 dez. 2019.

Ministério da Saúde. Secretaria de Atenção à Saúde. Política Nacional de Práticas Integrativas e Complementares no SUS. Brasília, DF: MS; 2006. (Série B. Textos Básicos de Saúde).

COSTA, C. G. A. et al. Hortas comunitárias como atividade promotora de saúde: uma experiência em Unidades Básicas de Saúde. Ciência \& Saúde Coletiva, v.20, n.10, p. 3099-3110, 2015.

CRAMER H., LAUCHE R., HALLER H., DOBOS G. A systematic review and meta-analysis of yoga for low back pain. The Clinical journal of pain, 2013. Disponível em: https://insights.ovid.com/article/00002508201305000-00010 Acesso em 14 abr 2020.

DOREEN, A. W.H. et al. Psychosomatic and physical responses to a multi-component stress management program among teaching professionals: A randomized study of cognitive behavioral intervention (CB) with complementary and alternative medicine (CAM) approach. Behaviour Research and Therapy; 80: 10-6, 2016.

FALKENBERG, R.I., EISING C., PETERS M.L. Yoga and immune system functioning: a systematic review of randomized controlled trials. J Behav Med 41, 467-482, 2018. Disponível em: https://link.springer.com/article/10.1007/s10865-018-9914-y Acesso em 14 abr 2020.

KIM, S. Y., SHIN, I. S., PARK, Y. J. Effect of acupuncture and intervention types on weight loss: a systematic review and meta-analysis. Obesity Reviews 19, 1585-1596, 2018. 
KUYKEN W., et al. Effectiveness and cost-effectiveness of mindfulness-based cognitive therapy compared with maintenance antidepressant treatment in the prevention of depressive relapse or recurrence (PREVENT): a randomised controlled trial. Lancet [Internet]. 2015. Disponível em: http://www.ncbi.nlm.nih.gov/pubmed/25907157 Acesso em 14 abr 2020.

KWON, C.Y. et al. Effectiveness and safety of ear acupuncture for trauma-related mental disorders after large-scale disasters. Medicine, 99:8, 2020.

LUZ, M. T. Cultura contemporânea e medicinas alternativas: novos paradigmas em saúde no fim do século XX. Physis: Revista de Saúde Coletiva, v. 7, n. 1, p. 13-43, jun. 1997.

LUZ, M. T. Pequenas destruições, danos irreparáveis: comentários aos modos sociais de vida no capitalismo pós - moderno; implicações na saúde. Revista EPOS, v. 4, n. 2, p. 01, 2013.

MERHY, E. E. Saúde : a cartografia do trabalho vivo. Hucitec: São Paulo, 2002.

RAMSDEN, A. Bate A. Using Word Clouds in Teaching and Learning. 2008.https://researchportal.bath.ac.uk/en/publications/using-word-clouds-in-teaching-and-learning (accessed 22 Jun2020).

SANTOS, F.A.S et al. Política de práticas integrativas em Recife: análise da participação dos atores. Revista de Saúde Pública. São Paulo, p. 1154-1159, 2011.

SOUSA, I. M. C.; BODSTEIN, R. C. A.; TESSER, C. D.; SANTOS, F. A. S.; HORTALE, V. A. Práticas integrativas e complementares: oferta e produção de atendimentos no SUS e em municípios selecionados. Cad. Saúde Pública vol.28 no.11 Rio de Janeiro Nov. 2012

SOUSA, I. M. C.; HORTALE, V. A.; BODSTEIN, R. C. A. Medicina Tradicional Complementar e Integrativa: desafios para construir um modelo de avaliação do cuidado. Ciênc. saúde coletiva, Rio de Janeiro, v. 23, n. 10, p. 3403-3412, Oct. 2018 Disponível em: <http://www.scielo.br/scielo.php?script=sci_arttext\&pid=S1413-

81232018001003403\&lng=en\&nrm=iso>. Acesso em 08 Apr. 2020.

TESSER, C. D.; SOUSA, I. M. C.; NASCIMENTO, M. C.. Práticas Integrativas e Complementares na Atenção Primária à Saúde brasileira. Saúde debate, Rio de Janeiro, v. 42, n. spe1, p. 174-188, Sept. 2018. Disponível em: <http://www.scielo.br/scielo.php?script=sci_arttext\&pid=S010311042018000500174\&Ing=en\&nrm=iso>. Acesso em 08 Apr. 2020.

TESSER, C. D.; SOUSA, I. M. C. Atenção Primária, Atenção Psicossocial, Práticas Integrativas e Complementares e suas Afinidades Eletivas. Saúde Soc. São Paulo, v.21, n.2, p.336-350, 2012.

VASCONCELOS, F. H.; ARAÚJO, G. C. Prevalence of chronic pain in Brazil: a descriptive study. Brazilian Journal Of Pain, v. 1, n. 2, p. 176-179, 2018.

VEEHOF M.M., OSKAM M.J., SCHREURS K.M.G., BOHLMEIJER E.T. Acceptance-based interventions for the treatment of chronic pain: a systematic review and meta-analysis. Pain [Internet]. 2011 Mar;152(3):53342. Disponível

em: http://www.embase.com/search/results?subaction=viewrecord\&from=export\&id=L51236091 Acesso em 28 mai 2020.

WHO. Estrategia de la OMS sobre medicina tradicional 2002-2005 (2002). Geneva: [s.n.]. Disponível em: https://www.paho.org/bra/index.php?option=com docman\&view=download\&alias=796-estrategiaoms-sobre-medicina-tradicional-2002-2005-6\&category slug=vigilancia-sanitaria-959\&/temid=965 
WHO. Traditional medicine strategy: 2014-2023. (2013). Geneva: [s.n.]. Disponível em: https://www.who.int/medicines/publications/traditional/trm strategy14 23/en/

WHO. Global Report on Traditional and Complementary Medicine 2019. Geneva: [s.n.]. Disponível em: http://www.who.int/traditional-complementary-integrative-medicine/en/

ZHANG Y., et al. The Effects of Traditional Chinese Exercise in Treating Knee Osteoarthritis: A Systematic Review and Meta-Analysis. PLoS One. 2017;12(1):e0170237. Published 2017 Jan 25. Disponível em: https://www.ncbi.nlm.nih.gov/pmc/articles/PMC5266306/ Acesso em 8 abr 2020. 